\title{
PRESENTACIÓN
}

\section{EDUCACIÓN SOCIAL EN PRISIONES. PLANTEAMIENTOS INICIALES Y POLÍTICAS ENCAMINADAS HACIA LA REINSERCIÓN DESDE LA PERSPECTIVA DE GÉNERO ${ }^{1}$}

\author{
Fanny T. Añaños-Bedriñana \\ Dpto. Pedagogía e Instituto de la Paz y los Conflictos, Universidad de Granada, España \\ Concepción Yagüe Olmos \\ Secretaría General de Instituciones Penitenciarias, Ministerio de Interior, España
}

\begin{abstract}
Reconocemos que un sistema de justicia penal eficaz, justo y humano se basa en el compromiso de proteger los derechos humanos en la administración de justicia y en la prevención del delito y la lucha contra la delincuencia.

(Declaración de Salvador, abril de 2010).
\end{abstract}

El ingreso en prisión constituye, habitualmente, una situación difícil y traumática para aquellos/as que son privados/as de su libertad. La dimensión y cantidad de personas inmersas en el contexto carcelario es grande, sin contar a los afectados/víctimas, a los profesionales e instancias relacionadas y a todos los componentes y colaboradores que intervienen en el sistema penitenciario; además de los recursos, medios, equipamientos, presupuestos, etc.

El contexto visibiliza un campo propio de la Pedagogía y de la Educación Social, con múltiples complejidades y fragilidades, escasa y parcialmente abordadas, pero que supone, a su vez, un escenario donde emergen fortalezas educativas, reeducativas y de reinserción que marcan algunos caminos a seguir. Todo ello evidencia la necesidad urgente de profundizar en los estudios y en las acciones penitenciarias, desde miradas socioeducativas y contemplando la perspectiva de género (cotidianamente olvidada). Este monográfico, que nace con el propósito de dignificar y mejorar las condiciones/calidad de vida a las personas que se hallan sumidas en este medio, nos facilita el camino para actuar en consecuencia.

Para acercarnos a la dimensión poblacional debemos recurrir a algunos datos, así en el panorama mundial el International Centre for Prison Studies de Londres elabora el llamado "World Prison Population List", que detalla la población penitenciaria genérica de 218 países independientes y territorios dependientes. La información más reciente corresponde a estudios elaborados en 2011 (Walmsley, 2012), de los que cabe citar que aproximadamente más de 10,1 millones de personas se hallan en instituciones penales en todo el mundo (preventivos y condenados), casi la mitad de ellas se concentran en Estados Unidos (2,29 m), Rusia (o,81 m) o China $(1,65 \mathrm{~m})$ sentenciados, a los que se suma 650.000 que están en "centros de detención" sin definir su situación 
legal). A estas cifras habría que añadir, también, a las personas internadas en distintos "centros" de privación de libertad que no figuran en las listas oficiales y de las que se desconoce prácticamente todo.

La misma fuente afirma que las poblaciones de reclusos/as crecen en los cinco continentes, así ha aumentado en el 78\% de los países (71\% África, 82\% América, 80\% Asia, 74\% Europa y 80\% Oceanía). En el caso de España la evolución marca que en 1990 había una población media interna de 33.058, en el 2000 creció a 45.104, en el 2010 se produce la etapa de mayor despunte con 73.929 (Ministerio de Interior, 2011) y, los datos más próximos, a enero de 2013, registran a 68.614 presos/as, de los que la mayoría son hombres (63.405) y el resto mujeres (5.209) (SGIP, 2013).

Pese a estas grandes cifras, se sabe poco de lo que acontece dentro del sistema y entre sus muros y aún perviven muchos problemas, entre otros, en los distintos niveles del sistema penitenciario: en los impactos y cotidianeidad de las vidas de los presos y presas, en los recursos, tratamientos y programas, en los procesos y métodos de intervención. Por ejemplo, en estos últimos apenas se conocen algunos seguimientos y menos sobre evaluaciones en cuanto a su eficacia y sus procesos de desarrollo (Del Pozo y Añaños-Bedriñana, 2013). Asimismo, cuestiones mucho más olvidadas son las acciones orientadas a promover la participación y motivación de los/as presos/as, hábitos y responsabilidades especialmente laborales o la escasa coordinación entre los procesos formativos y empleo (Elías, 2001).

Por su parte, otra cuestión pendiente y muy importante es la masificación y densidad poblacional dentro de casi todas las prisiones del mundo y España no es la excepción. A ello habría que sumar las distintas dificultades estructurales del sistema penitenciario, como la fuerte carga burocrática; la rémora de procedimientos y usos anclados en el siglo XX; la preminencia de los procedimientos estrictamente regimentales de prevención y seguridad sobre las acciones de intervención, que suponen a veces trabas insalvables para su ejecución; la falta de concienciación de una gran parte del personal penitenciario junto a la escasa formación en estas materias y descoordinación de los distintos estamentos, entre otros.

Sin embargo, pese a los puntos negros descritos y otros, existen numerosas actuaciones y posibilidades en el Tratamiento Penitenciario. La necesidad de intervenir para abordar la actuación y los procesos socioeducativos de inserción-reinserción social y la prevención de la reincidencia es una cuestión relativamente nueva. La acción en el medio penitenciario, en el lenguaje institucional, se denomina "Tratamiento", término que arrastra un nombre que es fruto de la herencia de los enfoques terapéuticos o clínicos prioritarios de la intervención, pero hoy sus contenidos son variados, término en el que se contemplan las cuestiones socioeducativas.

El Tratamiento es uno de los componentes con mayor relevancia en el sistema penitenciario español, pues así lo establece la constitución cuando dicta que las penas y medidas privativas de libertad deben de estar orientadas a la reeducación y reinserción social ${ }^{2}$. Todos los internos e internas, sin excepción, tienen derecho a participar en los programas de tratamiento, razón por la que la Administración se encuentra con la obligación de diseñar un Programa Individualizado (PIT), para cada uno de ellos, que permita resolver las distintas carencias y problemáticas que incidieron en su actividad delictiva. Pero está comprobado que la simple oferta no es suficiente, pues es preciso incentivarles de forma que intervengan en la planificación y ejecución del mismo si queremos que los programas sean seguidos con éxito. Por este motivo la actual regulación penitenciaria ha querido apartarse del enfoque clínico, a favor de una concepción más amplia del tratamiento bajo la denominación de intervención penitenciaria que cubre, no solo las actuaciones terapéuticas, sino las asistenciales, formativas, laborales o socioculturales.

Las políticas penitenciarias actuales son el fruto de un lento proceso de evolución, a partir de una legislación progresista con más de 30 años de recorrido desde la promulgación de la Ley Orgánica Penitenciaria de 1979:

1. En un primer momento, a finales de década de los años 80 , se apostó por una completa modernización de los establecimientos penitenciarios, clausurando paulatinamente los vetustos edificios heredados de la postguerra cuya principal finalidad era la contención. Esta trasformación nace con el "Plan de amortización

[ 8 ] FANNY T. AÑAÑOS-BEDRIÑANA, CONCEPCIÓN YAGÜE OLMOS

SIPS - PEDAGOGIA SOCIAL. REVISTA INTERUNIVERSITARIA [1139-1723 (2013) 22, 7-12] TERCERA ÉPOCA 
y creación de centros penitenciarios" aprobado en 1991 y que ha supuesto la creación de más de 30 nuevos centros (con la instauración del modelo residencial llamado "centro tipo") y el acondicionamiento de algunos de los ya existentes. Estas edificaciones son similares a pequeñas ciudades para albergar a cerca de 1.800 residentes, y permiten disponer de las más diversas infraestructuras: aulas, talleres, instalaciones deportivas, etc., con vistas a facilitar la implantación de acciones formativas de toda índole.

2. Paralelamente, desde mediados de los 90 se comenzó a promover la oferta y diversidad de actividades a disposición de los internos (bastante limitada hasta entonces), para cubrir todo un amplio espectro, desde la educación formal (que cubre los niveles iniciales hasta los estudios universitarios); los talleres ocupacionales, las actividades deportivas y de ocio y el trabajo productivo. Desde una primera etapa, en la que las políticas de intervención se definieron como la promoción de "la ocupación integral", procurando erradicar la inactividad, hasta la época actual en la que gracias a los fondos de cohesión europeos y a algunas fundaciones sociales se ha generado una gran oferta de acciones formativas, de talleres penitenciarios y de programas de acompañamiento para mejorar la empleabilidad e inserción laboral.

3. En esta última década han cobrado una enorme importancia las políticas a favor de nuevas formas de cumplimiento (en medio abierto y con medidas alternativas a la prisión). Paradójicamente, nuestra sociedad cada vez exige más seguridad y es menos tolerante hacia determinados comportamientos, de ahí que en las sucesivas reformas del Código Penal se hayan ido incluyendo nuevos tipos delictivos o el agravamiento de las sanciones para los existentes. A pesar de ello, en estos últimos años los responsables de las políticas penitenciarias están contrarrestando esta tendencia facilitando los programas que puedan desarrollarse en el medio libre y con asistencia a los recursos comunitarios, para aquellos internos con perfiles delictivos menos graves; potenciando los principios inspiradores del régimen abierto como son la atenuación de medidas de control, autorresponsabilidad del penado, normalización e integración social, evitación de la desestructuración familiar y coordinación con las instancias comunitarias de reinserción. En este proceso, los sistemas de ejecución de los países de nuestro entorno ya nos llevan un gran adelanto. Esta apuesta decidida a favor de nuevas formas de cumplimiento se sustenta en la creación de un importante número de nuevos Centros de Inserción Social -CIS- (modelo residencial en régimen de semilibertad) y la implantación de modernos sistemas de control telemático que permiten compatibilizar la condena con el desarrollo laboral y familiar prácticamente normalizado. Si bien es cierto que bajo la incidencia de la crisis económica el proceso de reintegración laboral se está ralentizando y ello obliga a reorientar las salidas hacia la potenciación de las acciones formativas en el exterior para que esta tendencia hacia el medio abierto no se resienta.

4. De forma más reciente la institución penitenciaria española ha apostado decididamente por instaurar la llamada cultura de la intervención psicosocial y el tratamiento específico, diseñando, implementando y evaluando complejos programas de tratamiento para ofertarlos a aquellos colectivos cuyas problemáticas personales, sociales o psicológicas están en la base de la comisión delictiva, bajo la constatación empírica de que la modificación de determinadas actitudes y factores tiene una clara incidencia en la seguridad pública, disminuyendo los índices de infracciones. En líneas generales, la mayoría de los programas actuales se sustentan en la teoría sobre la psicología criminal, denominada de riesgo-necesidades-responsividad de Andrews y Bonta (2006). Estos programas están enfocados hacia dos vertientes:

- Para aquellos colectivos concretos más vulnerables que precisan una atención específica, pues un trato igualitario no quiere decir que no puedan contemplarse determinadas características de un perfil de población que por su especial vulnerabilidad precisan una intervención diferente y un plus de atención para superar las dificultades que encuentran. En este caso se han implantado programas para jóvenes, extranjeros, discapacitados psíquicos, personas mayores -institucionalmente lo denominan "ancianos"- que permanecen en prisión y, para las mujeres encarceladas (mediante un programa de acciones para la igualdad, que intenta contrarrestar los factores de discriminación persistentes aún en el ámbito penitenciario). 
- Para atajar las situaciones y patologías sociales de mayor trascendencia en las actividades delictivas con programas específicos dirigidos a determinados colectivos que presenten unas características comunes ya sean estables o temporales: agresores sexuales o maltratadores en el ámbito familiar, enfermos mentales, drogodependientes, infractores de seguridad vial, internos en régimen cerrado, etc. Estas intervenciones específicas, de alta complejidad, beben de las más recientes y contrastadas teorías científicas y mantienen los estándares de calidad de los programas que se encuentran consensuados internacionalmente.

Como ejemplo de esta diversidad socioeducativa, citamos los principales programas específicos (número de centros penitenciarios donde se desarrollan -C-y media de participación -mp-), en el marco de la Administración General del Estado -AGE-3 (SGIP, 2011). Así, los programas más extendidos en el sistema son: "prevención de suicidios" (69 C y $328 \mathrm{mp}$ ), "módulos de respeto" (67 C y 15.726 mp), "discapacitados" (38 C y $557 \mathrm{mp}$ ) y "Unidades Terapéuticas" (34 C y $3.051 \mathrm{mp}$ ); por otro lado, los programas con vertiente de género destacados son "violencia de género" (35 C y $474 \mathrm{mp}$ ), "control de la agresión sexual" (32 C y $262 \mathrm{mp}$ ) y "Ser muje res" -atención a mujeres víctimas de violencia de género- (8 C y $85 \mathrm{mp}$ ).

En esta línea se ha iniciado la formación de un alto porcentaje de profesionales de la institución para acometer estas problemáticas, a la vez que se les ha dotado de los instrumentos y técnicas para su desarrollo (normalmente un manual que recoge tanto el marco teórico, como el desarrollo de las unidades y ejemplos variados de los ejercicios para diseñar cada una de las sesiones) ${ }^{4}$, instrumentos y escalas para la evaluación de la eficacia de estas actuaciones. Son programas intensos de larga duración (a veces uno o dos años), porque en el desarrollo del trabajo soportan un importante desgaste emocional, debido a que tienen que lidiar con una serie de situaciones que forman parte de la propia dinámica grupal: confrontaciones, resistencias, recaídas, enfrentamientos entre los miembros, desmotivaciones, abandonos, etc. Estas actuaciones se desarrollan en equipos multidisciplinares donde es frecuente que participen profesionales de organizaciones externas: universidades, organismos públicos y fundamentalmente $\mathrm{ONGs}^{5}$ y lo que resulta realmente interesante, es la paulatina implicación del personal de vigilancia de los centros, trascendiendo a las labores de mera custodia (tal como ocurre ya habitualmente con su integración ordinaria en los equipos de los módulos terapéuticos y en los módulos de respeto).

5. La propia idiosincrasia mediterránea española, que sacraliza el espacio público, conforma un régimen penitenciario donde la gran mayoría de los internados disponen de muchas horas de convivencia y de espacios temporales para actividades en común, en contraposición con los regímenes de nuestro entorno donde el aislamiento se rompe en muy pocas horas al día. En este sentido el objetivo que sustenta este ideal del tratamiento penitenciario sería el poder "encaminar la organización de los centros penitenciarios hacia espacios estrictamente socioeducativos donde cada persona ingresada encuentre la motivación, los medios y recursos necesarios para superar estas carencias o problemáticas específicas que contribuyeron a su actividad delictiva" $y$, en definitiva, evitar que la estancia de los internos e internas en los centros penitenciarios constituya un tiempo ocioso y perdido.

“Para garantizar el éxito de estas acciones se hace imprescindible crear previamente un ambiente seguro y ordenado, un sistema organizativo con normas de convivencia claras, previsibles, que minimice la tensión y reduzca las posibilidades de incidentes. Un sistema que enfatice las relaciones interpersonales positivas entre los internos y los miembros de personal, basadas en el respeto mutuo" (Yagüe, 2011, p. 4).

En el momento actual las políticas y objetivos estratégicos en los que la institución se encuentra más comprometida es en la creación de nuevos sistemas de organización y funcionamiento de la vida cotidiana en los propios establecimientos, expandiendo nuevas y pioneras iniciativas como los denominados módulos terapéuticos y los módulos de respeto, donde prima la implicación del interno en la propia organización y funcionamiento de la vida diaria del módulo. Por ello es un sistema diferente de "estar en la prisión y de vivir en la prisión", pues pasan de sentir que todo les viene impuesto (horarios o actividades) a percibir que

[ 10 ] FANNY T. AÑAÑOS-BEDRIÑANA, CONCEPCIÓN YAGÜE OLMOS

SIPS - PEDAGOGIA SOCIAL. REVISTA INTERUNIVERSITARIA [1139-1723 (2013) 22, 7-12] TERCERA ÉPOCA 
tienen capacidad para mejorar su calidad de vida en prisión y encuentran sentido a ocupar su tiempo en actividades por el valor intrínseco que tienen. Este enfoque tiene muchos elementos que pueden contribuir a mejorar su propia autoestima y facilitar su proceso de reinserción social.

En base a estos esfuerzos, distintos observadores externos no dudan en afirmar que el sistema penitenciario español, aún con sus fuertes carencias, puede considerarse como uno de los más avanzados del mundo, pues trata de ser coherente con el sentido que da a la pena de privación de libertad en su normativa legal y en respuesta a los principios de la Pedagogía Social. Pero en este camino no puede faltar el apoyo y compromiso de las instituciones comunitarias responsables de políticas sociales concretas y a las entidades de nuestro entorno geográfico implicadas en los objetivos en los que nos encontramos trabajando.

Por otro lado, todos los estudios que se han realizado sobre esta materia giran alrededor de la necesidad de actuar en el campo de la prevención de la comisión de delitos y de la reincidencia y, mucho mejor -y completa- si tenemos en cuenta la perspectiva de género en dicho marco. Hay indicios claros de que las estrategias de prevención del delito bien planificadas no solo previenen el delito y la victimización, sino que también promueven la seguridad de la comunidad y contribuyen al desarrollo sostenible de los países (ONU, 2007). En esa línea, todo indica que las políticas responsables que dan resultados positivos en relación a la prevención del delito mejoran la calidad de la vida de todos los ciudadanos, a su vez, producen beneficios a largo plazo al reducir los costos relacionados con el sistema formal de justicia penal, así como otros costos sociales y personales resultantes de la acción delictiva. También dan la oportunidad de ofrecer programas con enfoques más educativos y sociales a los problemas de la delincuencia.

En el presente monográfico, bajo el título "Educación Social en Prisiones", desde nuestra revista bandera, se esbozan planteamientos, realidades, experiencias y propuestas críticas que a continuación presentamos. Además, procuraremos mirar los temas desde el enfoque de género, porque queremos visibilizar el hecho de que las mujeres -que representan porcentajes menores de población tanto en datos nacionales como internacionales-, perviven en una situación aún más lamentable e invisibilizada; en las que las repercusiones de la entrada a prisión son más negativas (para ellas mismas, para sus hijos/as y sus familias) y, es fácil constatar que están más expuestas y son atendidas con menor grado de calidad en el sistema penitenciario.

El trabajo de Fanny T. Añaños-Bedriñana, Ma Pilar Sánchez Fernández y Juan José Llópiz Llácer “Aproximación a los contextos en prisión. Una perspectiva socioeducativa", como punto de partida sitúa y contextualiza el medio penitenciario, para conocer sus dinámicas, organizaciones, formas y procedimientos de intervención; así como analiza los diversos regímenes de vida establecidos en relación a los espacios, tiempos y las consecuencias del encarcelamiento. Por su parte, Gilles Chantraine y Nicolas Sallée nos ofrecen "La educación puesta a prueba en un espacio de reclusión", artículo que se adentra en los establecimientos penitenciarios para menores en Francia, centrando el foco en las diferencias de las lógicas de la seguridad y de la educación observadas en las prácticas tanto de educadores como de vigilantes de prisión.

"El ambiente en prisión: La atención recibida por las reclusas y las relaciones intramuros" es el trabajo de María del Mar García-Vita y Miguel Melendro Estefanía. Profundizan en dinámicas internas dentro de la prisión, analizando el ambiente relacional, apoyos y la atención recibida durante el cumplimiento de condena, llegando a identificar factores de protección que se encaminan a facilitar la reinserción. Cierra el bloque Francisco del Pozo Serrano, Francisco Jiménez Bautista y Ángel Turbi Pinazo con el artículo "El tratamiento con mujeres: actuación socioeducativa y sociolaboral en prisiones", en el que estudian la intervención penitenciaria a través de los programas socioeducativos y sociolaborales, visibilizando las valoraciones de las participantes, los vacíos, debilidades y fortalezas de los programas.

Esperamos que estos aportes nos ayuden a configurar y ampliar la temática penitenciaria, que en periodos de larga duración o coyunturales, se abstrajeron y abstraen en un sinnúmero de procesos políticos, sociales, económicos, ideológicos, etc., y que en el campo de la educación, salvo excepciones, han sido poco significativos. También, esperamos estar contribuyendo a atraer, motivar, reflexionar críticamente, estudiar, producir, socializar y actuar, desde los escenarios académicos, profesionales, administrativos y de todas las personas involucradas $y / 0$ interesadas. 
Finalmente, agradecemos a los/as magníficos/as y expertos/as colaboradores/as de este monográfico por sus propuestas valiosas, a Pedagogía Social. Revistas Interuniversitaria que ha hecho una apuesta valiente al dar luz a este ámbito de la Educación Social y, muy especialmente, a todas las personas internadas que de una u otra forma han dado sus testimonios o son el objeto de nuestras reflexiones.

\section{Referencias bibliográficas}

Andrews, D. y Bonta, J. (2006). The Psychology of Criminal Conduct (4a ed.). Cincinnati (EEUU): Anderson Publishing Co.

Del Pozo, F.J. y Añaños-Bedriñana, F.T. (2013). La Educación Social Penitenciaria: ¿De dónde venimos y hacia dónde vamos? Revista Complutense de Educación, 24,1, 47-68.

Elías Ortega, A. (2001). El trabajo de los presos dentro de las cárceles. En C. Manzanos Bilbao (Coord.). Cárcel, Drogas y Sida. Trabajo Social Frente al Sistema Penal. Bilbao: Salhaketa. Pp. 37-86. Recuperado de http://www.ikusbide.org/ data/documentos/LIBROCAR2000.pdf

Ministerio de Interior (2011). Anuario Estadístico del Ministerio de Interior. Asuntos Penitenciarios 4. Madrid: Ministerio de Interior / SGIP.

Oficina de las Naciones Unidas contra la Droga y el Delito (2011). Sección de Justicia. Prevención del Delito y Reforma de la Justicia Penal. Viena: UNODC.

ONU (2007). Recopilación de reglas y normas de las Naciones Unidas en la esfera de la prevención del delito y la justicia penal. Nueva York: Oficina de las Naciones Unidas contra la Droga y el Delito.

SGIP (2011). Subdirección General de Tratamiento y Gestión. Datos relativos al cuarto trimestre de 2011.

SGIP (2013). Estadística penitenciaria. Recuperado Recuperado de http://www.institucionpenitenciaria.es/ web/portal/documentos/estadisticas.html

Walmsley, R. (2012). World Prison Population List (ninth edition). London: International Centre for Prison Studies.

Yagüe Olmos, C. (2011). Objetivos estratégicos del Tratamiento Penitenciario. Las Políticas de intervención. Tratamiento y Seguridad. Madrid: Máster Universitario en Administración y Gestión de Centros Penitenciarios. UNED. Plataforma formativa virtual ALF.

\section{Notas}

1 Este monográfico tiene como marco la convocatoria de proyectos |+D+| del Plan Nacional de Investigación, "Mujeres reclusas drogodependientes y su reinserción social. Estudio socioeducativo y propuestas de acción“ [Ref. EDU2009-13408], financiado por el Ministerio de Economía y Competitividad (MICCIN) del gobierno español.

2 Declaración taxativa del artículo 25 de la Constitución Española de 1978.

3 Administración General del Estado -AGE-. Está compuesta por todas la Comunidades Autónomas a excepción de Cataluña, que tiene las competencias en materia penitenciaria transferidas.

4 Manuales que bajo el epígrafe de "Documentos Penitenciarios" pueden encontrarse a disposición en: http://www.institucionpenitenciaria.es/web/portal/documentos/publicaciones.html

5 A pesar de que un importante número de ellas está haciendo ímprobos esfuerzos por mantener esta colaboración, hay que constatar que a tenor de los recortes en políticas sociales, la gran difusión de colaboraciones de las ONGs de la última década está decayendo muy significativamente por falta de subvenciones. Por este motivo la mayoría de estos programas se mantienen con el impulso de los propios profesionales penitenciarios y abriendo nuevas vías de colaboración, fundamentalmente con universidades y centros formativos.

[ 12 ] FANNY T. AÑAÑOS-BEDRIÑANA, CONCEPCIÓN YAGÜE OLMOS

SIPS - PEDAGOGIA SOCIAL. REVISTA INTERUNIVERSITARIA [1139-1723 (2013) 22, 7-12] TERCERA ÉPOCA 